LUIS A. CORDERO

MARISA FERNÁNDEZ

MANUEL DE LEÓN

MARTÍN SARALEGUI

\title{
Compact symplectic four solvmanifolds without polarizations
}

Annales de la faculté des sciences de Toulouse $5^{e}$ série, tome 10, $\mathrm{n}^{\mathrm{o}} 2$ (1989), p. 193-198

<http://www.numdam.org/item?id=AFST_1989_5_10_2_193_0>

(C) Université Paul Sabatier, 1989, tous droits réservés.

L'accès aux archives de la revue «Annales de la faculté des sciences de Toulouse » (http://picard.ups-tlse.fr/ annales/) implique l'accord avec les conditions générales d'utilisation (http://www.numdam.org/conditions). Toute utilisation commerciale ou impression systématique est constitutive d'une infraction pénale. Toute copie ou impression de ce fichier doit contenir la présente mention de copyright.

\section{Numdam}

Article numérisé dans le cadre du programme

Numérisation de documents anciens mathématiques

http://www.numdam.org/ 


\title{
Compact Symplectic Four Solvmanifolds Without Polarizations
}

\author{
Luis A. Cordero(1), Marisa Fernández ${ }^{(2)}$, \\ Manuel De León ${ }^{(3)}$, Martín Saralegui ${ }^{(3)}$
}

RÉsumé. - On obtient une famille $M_{\lambda}(k)$ de variétés symplectiques compactes de dimension 4. La variété $M_{\lambda}(k)$ est l'éclatement d'une variété compacte, résoluble et symplectique $M^{4}(k)$. La variété $M^{4}(k)$ ne posséde aucune structure complexe (donc kählerienne) mais elle a toutes les propriétés d'une variété kählerienne. Alors $M^{4}(k)$ ne posséde aucune polarisation totalement complexe ni kählerienne. En outre $M_{\lambda}(k)$ ne posséde aucune polarisation avec index différent de zero.

ABSTraCt. - In this paper a class of compact 4-dimensional symplectic manifolds $M_{\lambda}(k)$ is obtained by blowing up a certain compact symplectic solvmanifold $M^{4}(k)$ at $\lambda$ distinct points. Although $M^{4}(k)$ has all the topological properties of a Kähler manifold it has no complex (and hence no Kähler) structures; therefore, $M^{4}(k)$ has no totally complex (and hence no Kähler) polarizations. Moreover, we prove that $M_{\lambda}(k)$ has no polarizations with non-zero real index.

\section{Introduction}

In order to quantizate a symplectic manifold, three additional structures are needed : a prequantization, a polarization, and a metaplectic frame bundle. Thus, the existence of symplectic manifolds which do not admit polarizations has significant implications for geometric quantization theory. In fact, few examples of such manifolds are known. For instance, $S^{2} \times S^{2}$ has no polarizations with non-zero real index, but it admits a Kähler

(1) Departamento de Geometría y Topología, Faculdad de Matemáticas, Universidad de Santiago de Compostela, 15705 Santiago de Compostela (Spain)

12) Departamento de Matemáticas, Faculdad de Ciencias, Universidad del País Vasco, Apartadi 644, Bilbao (Spain)

(3) C.E.C.I.M.E., C.S.I.C., Serrano 123, 28006 Madrid (Spain) 
Luis A. Cordero, Marisa Fernández, Manuel de León, Martín Saralegui

polarization (see $[9,11])$. On the other hand, a symplectic manifold carries totally complex (resp. Kähler) polarizations (that is, with zero real index) if and only if it admits compatible complex (resp. Kähler) structures. Therefore, the manifolds $E^{4}$ of [3] (which are circle bundles over circle bundles over a torus $T^{2}$ ) with first Betti number 2 or 3 have no Kähler polarizations and, moreover, if $b_{1}\left(E^{4}\right)=2$, then they have no totally complex polarizations. But all of these symplectic manifolds often have real polarizations.

Recently, Gotay [6] described a class of symplectic 4-manifolds which do not admit polarizations of any type whatever. These manifolds are constructed by repeatedly blowing up $E^{4}$ with $b_{1}\left(E^{4}\right)=2$. This construction has been extended by M. Fernández and M. de León [5] by considering circle bundles over circle bundles over a Riemann surface of genus $g>1$.

In this paper, following Gotay's construction, a class of compact 4dimensional symplectic manifolds $M_{\lambda}(k)$ is obtained by blowing up a certain manifold $M^{4}(k)$ at $\lambda$ distinct points. Here $M^{4}(k)$ is a compact symplectic solvmanifold constructed in [4]. Although $M^{4}(k)$ has all the topological properties of a Kähler manifold it has no complex (and hence no Kähler) structures (see [4] for the details); therefore, $M^{4}(k)$ has no totally complex (and hence no Kähler) polarizations. Moreover, we prove that $M_{\lambda}(k)$ has no polarizations with non-zero real index.

We don't know if $M_{\lambda}(k)$ admits or not totally complex polarizations; if they do, this fact would be very interesting for the Kählerian Geometry realm because they would provide, using [8], new examples of compact Kähler manifolds.

\section{Geometric Quantization}

First, let us recall some well-known facts about the theory of geometric quantization (for more details, see $[10,12,13]$ ).

Let $(X, \omega)$ be a $2 n$-dimensional symplectic manifold. The supplementary structures on $X$ needed for geometric quantization are the following :

(1) A prequantization of $(X, \omega)$, that is, a complex line bundle $L$ over $X$ with a connection $\nabla$ such that the connection form $\alpha$ satisfies the prequantization condition

$$
\begin{gathered}
d \alpha=-(h)^{-1} \omega, \\
-194-
\end{gathered}
$$




\section{Compact Symplectic Four Solvmanifolds Without Polarizations}

where $h$ is Planck's constant. Further on, we shall suppose that there also exists a $\nabla$-invariant Hermitian structure $<,>$ on $L$.

(2) A polarization of $(X, \omega)$, that is, an involutive $n$-dimensional complex distribution $F$ on $X$ such that

$$
\omega^{\mathbf{C}}{ }_{\mid(F \times F)}=0
$$

and $\operatorname{dim}(F \cap \bar{F})$ is constant, where $\bar{F}$ denotes the complex conjugate of $F$.

A polarization $F$ defines two complex distributions $F \cap \bar{F}$ and $F+\bar{F}$ on $X$ which are the complexifications of certain real distributions $D$ and $E$, respectively :

$$
F \cap \bar{F}=D^{\mathbf{C}} \text { and } F+\bar{F}=E^{\mathbf{C}} .
$$

(Note that $D$ is the $\omega$-orthogonal complement of $E$.) Since $F$ is involutive $D$ is too, so that $D$ defines a foliation on $X$. Let $X / D$ be the space of leaves of $D$ and $\pi_{D}: X \longrightarrow X / D$ the canonical projection.

A polarization $F$ is strongly admissible if $E$ is involutive, the spaces of leaves $X / D$ and $X / E$ are quotient manifolds of $X$ and the canonical projection $\pi_{E D}: X / D \longrightarrow X / E$ is a submersion. The dimension $d$ of $D$ is called the real index of $F$. When $d=n, F=\bar{F}$ and $F$ is said to be $a$ real polarization. Then $D=E=F \cap T X$.

Now, let $J$ be an almost complex structure on $X$ determined by $\omega$ (see [12]). Then, there is a Lagrangian splitting $T X=D \oplus J D$ so that $(T X, J)$ may be identified with $D^{\mathrm{C}}$. As a consequence, it follows that the odd real Chern classes of $(T X, J)$ vanish.

On the other hand, when $d=0, F$ is said to be a totally complex polarization. Then $F \cap \bar{F}=0, E=T X$ and $F$ determines an almost complex structure $J$ on $X$, which is actually a complex structure because $F$ is integrable (see [12]). Moreover, since $\omega(J u, J v)=\omega(u, v)$ for all $u, v \in T X$, we can define an Hermitian metric $<,>$ on $X$ by $\langle u, v\rangle=\omega(u, J v)$. If $<,>$ is positive definite then $(X, J,<,>)$ is a Kähler manifold and $F$ is said to be Kähler. Then a symplectic manifold $(X, \omega)$ carries totally complex (resp. Kähler) polarizations if only if it admits compatible complex (resp. Kähler) structures.

(3) A metaplectic structure on $X$, that is, a right principal $M p(n, \mathbf{R})-$ bundle over $X$, where $M p(n, \mathbf{R})$ is the metaplectic group (the double covering of the symplectic group $\left.S_{p}(n, \mathbf{R})\right)$. The metaplectic structure is used 
to define the complex line bundle $\sqrt{\Lambda^{n} F}$, the bundle of half-forms relative to $F$. This bundle has a canonically defined partial flat connection.

Then the elements of the quantum state space $H$ corresponding to the geometric quantization structures given above are sections of the complex line bundle $L \otimes \sqrt{\Lambda^{n} F}$ which are covariantly constant along $F$. If $F$ is strongly admissible then the wave functions are represented by sections of $L \otimes \sqrt{\Lambda^{n} F}$ which are covariantly constant along $D$ and holomorphic along the fibers of $\pi_{E D}$. Such sections have supports contained in the subset $S$ of $D$ which is the union of those leaves of $D$ for which the holonomy group of the induced flat connection in $L \otimes \sqrt{\Lambda^{n} F}$ is trivial. The set $S$ is called the Bohr-Sommerefeld variety, since it is locally determined by the generalized Bohr-Sommerefeld conditions. Each leaf of $D$ has a canonically defined parallelization. When $F$ is strongly admissible and complete (that is, the leaves of $D$ are complete manifolds) it is possible to decompose $S$ as follows:

$$
S=\bigcup_{a=0}^{d} S_{a}
$$

where $S_{a}$ is the union of all those leaves of $D$ contained in $S$ which are affinely isomorphic to the cylinder $T_{a} \times \mathbf{R}^{d-a}$. Thus $\operatorname{dim} S_{a}=2 n-a$.

\section{The manifolds $M_{\lambda}(k)$}

First we recall some facts about the manifolds $M^{4}(k)$ of [4]. The space $M^{4}(k)$ is the product manifold $X(k) \times S^{1}$, where $X(k)$ is the compact 3solvmanifold $S_{1} / D_{1}$ considered in [1, p. 20]AGH, $S_{1}$ being the 3 -dimensional solvable non-nilpotent Lie group of matrices of the form

$$
\left(\begin{array}{cccc}
e^{k z} & 0 & 0 & x \\
0 & e^{-k z} & 0 & y \\
0 & 0 & 1 & z \\
0 & 0 & 0 & 1
\end{array}\right)
$$

where $x, y, z \in \mathbf{R}$, and $D_{1}$ being a discrete subgroup of $S_{1}$ such that the quotient space $S_{1} / D_{1}$ is compact. The spaces $M^{4}(k)$ have symplectic structures but can have no complex structures. The key for this is Yau's Theorem 2 in [14].

Next, we prove that $X(k)$ can be seen as the bundle space of a 2-torus bundle over the circle $S^{1}$. Let $\rho: \mathbf{Z} \longrightarrow D$ iff $\left(T^{2}\right)$ be the representation 
defined by $\rho(m)=[A(m)]$, where $[A(m)]$ represents the transformation of $T^{2}$ covered by the linear transformation of $\mathbf{R}^{2}$ corresponding to the matrix

$$
A(m)=\left(\begin{array}{cc}
e^{k m} & 0 \\
0 & e^{-k m}
\end{array}\right)
$$

Now, $\rho$ induces a representation $\rho^{\prime}: \mathbf{Z} \longrightarrow \operatorname{Diff}\left(\mathbf{R} \times T^{2}\right)$ as follows $: \mathbf{Z}$ operates on $\mathbf{R}$ by covering transformations, and on $T^{2}$ by $\rho$. Then we have a bundle structure for $X(k)$ over $S^{1}$ with fibre $T^{2}$, that is

$$
X(k) \cong \mathbf{R} \times \mathbf{z} T^{2} .
$$

Now, blow up $M^{4}(k)$ at $\lambda$ distinct points using the technique of Gromov and McDuff (see [7]). The resulting manifolds $M_{\lambda}(k)$ are compact 4manifolds diffeomorphic to $M^{4}(k) \# \lambda \overline{\mathbf{C P}}^{2}$, where $\overline{\mathbf{C P}}^{2}$ denotes $\mathbf{C P}^{2}$ with the reversed orientation. Then $M_{\lambda}(k)$ has signature $\sigma\left(M_{\lambda}(k)\right)=-\lambda$ and Betti numbers

$$
\begin{aligned}
& b_{0}\left(M_{\lambda}(k)\right)=b_{4}\left(M_{\lambda}(k)\right)=1, \\
& b_{1}\left(M_{\lambda}(k)\right)=b_{3}\left(M_{\lambda}(k)\right)=2, \\
& b_{2}\left(M_{\lambda}(k)\right)=2+\lambda .
\end{aligned}
$$

Thus, the Euler number of $M_{\lambda}(k)$ is $\chi\left(M_{\lambda}(k)\right)=\lambda$.

Proposition 1..- The manifolds $M_{\lambda}(k)$ have symplectic structures.

Proof.-This is a direct consequence of [7, proposition 3.7]McD.

Finally, we prove the main result :

Theorem 1.. - The symplectic manifolds $M_{\lambda}(k)$ have no polarizations of nonzero real index $d$.

Proof. - We shall only consider two cases, depending upon the value of the real index $d, 1 \leq d \leq 2$.

$\underline{l=1}:$ In this case $D$ would define a field of line elements on $M_{\lambda}(k)$. But this is impossible since $\chi\left(M_{\lambda}(k)\right)=\lambda \neq 0$. 
$\underline{d=2}:$ In this case the first real Chern class of $\left(T M_{\lambda}(k), J\right)$ must vanish. But we have

$$
\mathbf{c}_{1}^{2}\left(T M_{\lambda}(k), J\right)=3 \sigma\left(M_{\lambda}(k)\right)+2 \chi\left(M_{\lambda}(k)\right)=-\lambda \neq 0 .
$$

\section{Références}

[1] Auslander (L.), Green (L.), Hahn (F.). - Flows on Homogeneous Spaces. Annals of Math. Studies 53, Princeton Univ. Press, 1963.

[2] Deligne (P.), Griffithis (P.), Morgan (J.), Sullivan (D.).- Real homotopy theory of Kähler manifolds. Invent Math. 29, 1975, p. 245-274.

[3] Fernández (M.), Gotay (M.J.), Gray (A.). - Compact parallelizable four dimensional symplectic and complex manifolds. Proc. Amer. Math. Soc. 103, 1988, p. 1209-1212.

[4] Fernández (M.), Gray (A.). - Compact symplectic four dimensional solvmanifolds not admitting complex structures. (Preprint).

[5] Fernández (M.), de LEÓN (M.). - Compact symplectic four dimensional manifolds no admitting polarizations. (Preprint).

[6] Gotay (M.J.).- A class of non-polarizable symplectic manifolds. Monatshefte für Math. 103, 1987, p. 27-30.

[7] Mc DUFF (D.). - Examples of simply connected symplectic non-Kählerian manifolds. J. Diff. Geometry 20, 1984, p. 267-277.

[8] Kodaira (K.).- - On the structure of compact complex analytic surfaces, I. Amer. J. Math. 86, 1964 , p. 751-798.

[9] Simms (D.J.). - Geometric quantization of energy levels in the Kepler problem. Symp. Math. 14, 1974, p. 125-137.

[10] SNIATYCKI (J.).- Geometric Quantization and Quantum Mechanics. Applied Mathematical Sciences Series 30, Springer-Verlag, New York, 1980.

[11] VaIsman (I.). - The Bott obstruction to the existence of nice polarizations. Mh. Math. 92, 1981 , p. 231-238.

[12] Weinstein (A.). - Lecture on Symplectic Manifolds. CBMS Reg. Conf. Ser. Math. 29, Amer. Math. Soc., Providence, R.I., 1977.

[13] IVoodhouse (N.M.J.). - Geometric Quantization. Clarendon Press, Oxford, 1980.

[14] YAU (S.T.). - Parallelizable manifolds without complex structure. Topology 15, 1976 , p. 51- 53 . 